\title{
Non-Model Based Method for an Automation of 3D Acquisition and Post-Processing
}

\author{
B. Loriot ${ }^{*+}$ and R.Seulin* and P. Gorria* \\ * Le2i CNRS UMR5158, 12 rue de la Fonderie, 71200 Le Creusot, France \\ + Dynamic3D, 4 rue de la Mare, 71530 Crissey, France
}

Received $16^{\text {th }}$ May, 2008; accepted $22^{\text {nd }}$ December,2008

\begin{abstract}
Most of the automation for 3D acquisition concerns objects with simple shape, like mechanical parts. For cultural heritage artefacts, the process is more complex, and it doesn't exist general solution nowadays. This paper presents a method to generate a complete 3D model of cultural heritage artefacts. In a first step, MVC is used to solve the view planning problem. Then, holes remaining in 3D model are detected, and their features are calculated to finish acquisition. Different post-processing are applied on each view to increase quality of the 3D model. This procedure has been tested with simulated scanner, before being implemented on a motion system with five degrees of freedom.
\end{abstract}

Key Words: 3D acquisition, cultural heritage artefacts, scanners automation, view planning

\section{Introduction}

The demand for high-quality three dimensional (3D) models of complex objects is growing in a wide range of applications (e.g., industrial [1], medical [2-3-4-5-6], cultural [7-8-9], architectural [10-11], ...). Numerous applications require the computer vision approach to object surface reconstruction. These applications relate to fragile objects whose handling is impossible. Only non-contact sensors are usable.

3D acquisition devices collect 3D coordinates of an object surface [12-13]. To acquire the shape from all sides, many scans are necessary. Our system is based on several assumptions: the scanner remains calibrated between each acquisition and the object size and material is compatible with the scanner specifications. For each scan, post-processing is required (noise removing, segmentation, registering, meshing, smoothing and mesh cleaning). Each scan captures a portion of the shape of the object, and in order to merge all of the scans into a single shape we must place them in the same coordinate system. If there is a lack of data, others scans may be necessary. 3D acquisition and post-processing are long and the experience of the user has a significant influence on the resulting 3D model quality.

Acquisition and post-processing need to be automated in order to reduce human operator influence and speed up the measurement process. The NBV algorithms have for objective to calculate this set of positions necessary for the digitization. These algorithms can be divided in two categories : model based methods (when CAD model or coarse model is available) and non model based methods (when no a priori knowledge of the shape of the object is available). For cultural heritage artefacts, we must use non-model based methods. The main objective of the proposed method is to automatically reach maximum surface coverage.

Correspondence to: benjamin.loriot@u-bourgogne.fr

Recommended for acceptance by David Fofi and Ralph Seulin

ELCVIA ISSN: 1577-5097

Published by Computer Vision Center / Universitat Autonoma de Barcelona, Barcelona, Spain 
Proposed method is based on view planning methods, described in section 2. This proposed method is multi-phase approach (coarse to fine) and used a triangulation based laser scanner (Vi910 from Konica Minolta [14]). Two techniques are used: the first is based on Mass Vector Chain (MVC), presented in the section 3, and allows to create a coarse model. The second uses the "holes" (lack of data) in the mesh to define the next best viewpoint, by calculating "position", "normal" and "size" of each hole remaining in the coarse model. The process is fully described in section 4. Applications on cultural heritage artefacts and results are shown in section 5 .

\section{View planning}

To acquire the complete surface of a 3D object using triangulation-based laser scanner, several range images from different viewpoints are necessary. To define these positions, we have to find a solution to the next best view (NBV) problem. To solve this problem, we have studied different methods of view planning (or sensor planning).

View planning is used to minimize the number of required views to reconstruct a complete 3D model or to ensure that the viewpoints selected are as close as possible to the optimal viewpoints. Some algorithms solve the "next best view" (NBV) problem in order to determine the next position for the range scanner given its previous scans of the object. Several algorithms are described by Prieto in [15] or Scott \& al. in [16]. These methods can be classified in two categories : model-based method (Cowan \& al. [17], Scott \& al. [18]) and non-model based method (Connolly [19], Banta \& al. [20][21], Maver \& al. [22], Pito \& al. [23], Yuan [24]).

In [17], Cowan \& al. show a method to automate the determination of the location where a non-contact sensor may be placed to observe one or more object surfaces. They identify several requirements that affect sensor placement : resolution, focus, field of view, visibility, view angle, prohibited region. For each constraint, a 3D region of viewpoint is defined. The intersection of these region define a new 3D region where the sensor can be positioned.

In [18], Scott \& al. use a mutli-phase approach to solve the NBV problem (Fig.1). In the first step, a preprogrammed scan is performed to produce a polygonal mesh "rough model", an approximation of the object geometry. Next the rough model is decimated to a sampling resolution just adequate for view planning. The rough model is then used to develop a view plan for a subsequent stage of precision measurement in compliance with the model specification. Then, the rough model is segmented into a set of surfaces based on the different surface features (cavity, hole, ...). A measurability matrix (surface point and viewpoints) is computed for each segmented region.

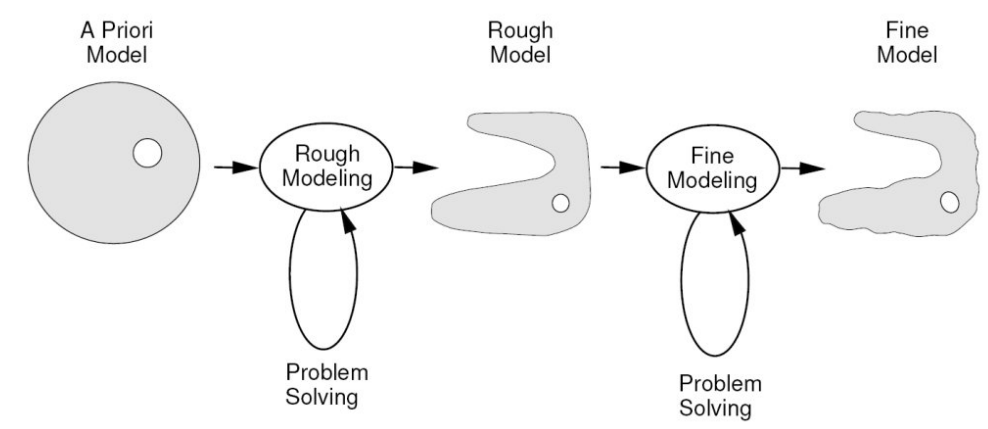

Fig.1: Multi-stage Model-based View Planning

One of the earliest papers on view planning was by Connolly. He has been the first to be used the term "next-best-view". In [19], he shows a volume based method. He describes algorithms for finding a set of covering views using the octree data structure (Fig.2). This octree contains four node types : Parent (nodes which have children), Empty (empty space which is visible from at least one of the views used to generate the octree), Occupied (they contain points on the object's surface) and Unseen (volumes which have not yet been seen by any view). 


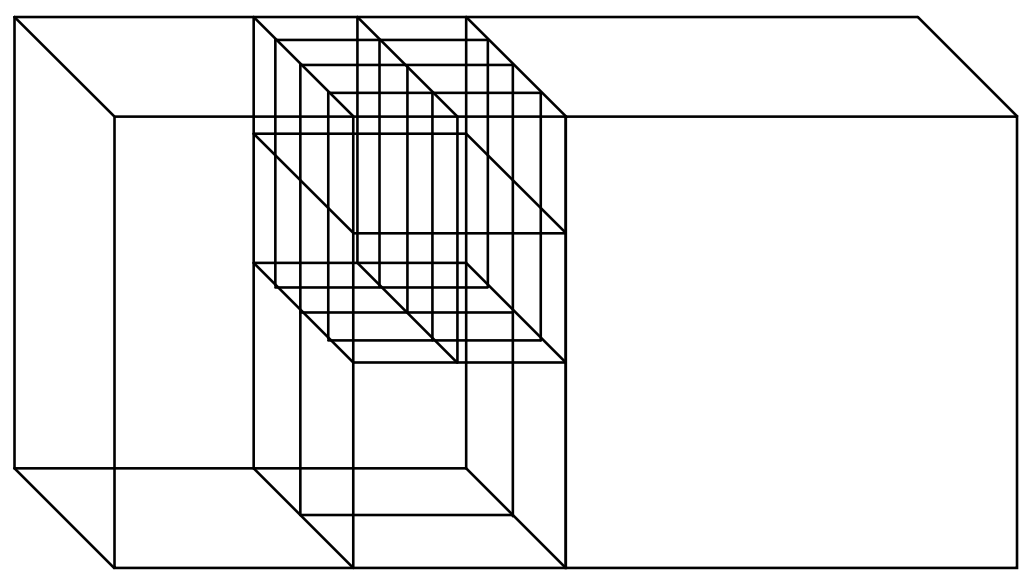

Fig.2: Octree structure

With this structure and an algorithm which produces a single view vector indicating the next location for a sensor, the following step are used to complete the octree model :

1. pick an arbitrary view vector to start with

2. assimilate the most recently selected view in the octree

3. determine the next view vector

4. if no such vector exists, terminate, else go back to 2

The first described algorithm (Planetarium Algorithm) uses a sphere which is sampled along latitude and longitude. At each sample point, a hidden-line view of the octree is generated considering only Occupied and Unseen nodes. Following this, the area of Unseen nodes covering the viewplane is determined. This value provides a measure of how much of the unknown area can be eliminated by taking a view from each point, and takes into account occlusion by other Unseen and Occupied nodes. The viewpoint with the largest unseen area is selected as the NBV.

The second algorithm (Normal Algorithm) uses information about the faces in an octree which are common to both Unseen and Empty nodes. Such faces will be referred to as Unseen /Empty faces. In effect, this algorithm measures the visibility of the Unseen nodes within the tree. The algorithm determines the number of faces of each Unseen node that are exposed to the region of space which is known to be empty. This is accomplished by recording the areas of Unseen / Empty faces. Six separate area sums are kept, since the faces can be oriented in any of six directions. It is faster but does not deal as well with self-occluding scenes.

In [20], Banta \& al. use a 3D occupancy grid to represent the object model. This 3D grid is composed of a matrix of points marked as occupied or unoccupied. An occupied voxel represents a cubic volume within the 3D imaging domain which is intersected by an arbitrary solid. The 3D model of a scene can be realized by determining the state of each voxel from one or a series of range images. An occupancy grid can be used to construct an object model up to any specified resolution. A model can be constructed by registering disjoint or overlapping occupancy grids into a world database. This allows reconstruction of a large and complex model from a set of occupancy grids. Only the camera pose is required to register an occupancy grid with a world database.

Several issues must be addressed in mapping the range data to 3D (Fig.3):

1. The occupancy grid must be correctly aligned with the range image so that the information contained in both may be combined.

2. A scaling transformation must be applied to the data to ensure that it is the correct size. 


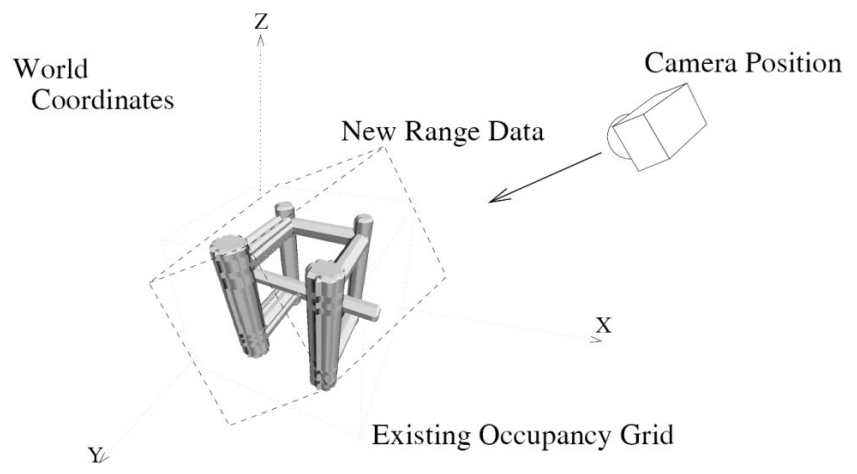

Fig.3: The range information must be correctly aligned before assimilation into a world model

The points which are blocked by the mapped surface points are identified by extrapolating along rays connecting the range sensor and the detected surface points. Once the location of the hidden points is determined, a temporary occupancy grid will be created, assuming that all points not currently visible are occupied. During this phase, a surface occupancy grid is built (occupancy grid containing only surface information). This new grid is used for determining the next-best-view.

The next sensor orientation is computed with the jump edges, the singularities normal to the view direction which might be occluding additional scene information. For the next sensor position, a sphere with a radius depending of the size of the occupancy grid is created. The position is defined by the intersection of this sphere and the sensor orientation.

In [21], Banta \& al. describe a algorithm with three phases according to the number of views. The three methods used are:

1 edge based sensor placement described in [20]

2 targeting the occluded surface centroid: in this approach, the reconstructed voxel model is examined. The new view points toward the view sphere center and is oriented on the voxel face closest to the centroid of the occluded region. This method give good results when there is one continuous occluded surface.

3 clustering the occluded surface data: the occluded patches are divided into an unspecified number of areas. Then, the next sensor position points toward the view sphere center and is oriented on the voxel face closest to the mean of the largest cluster found by the clustering algorithm.

In [22], the approach of Maver \& al. was conceived for a long baseline laser profile scanner with a positioning system limited to a single rotational degree of freedom. The two-stage method separately considered source and receiver occlusions:

1. camera occlusion: it arises when a part of the illuminated surface in the scene is occluded to the camera by another part of the scene (Fig.4c).

2. light occlusion: it arises when the direct laser light does not reach a part of the surface in the scene because it is reflected from another part of the scene (Fig.4b).

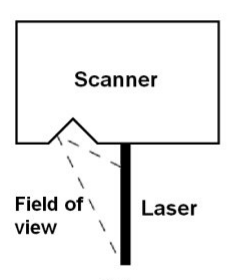

(a)

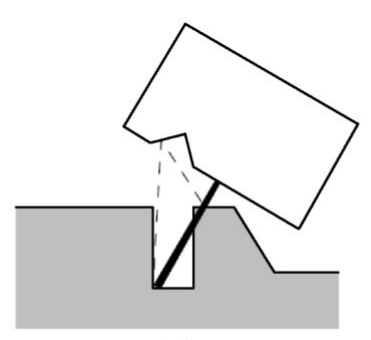

(b)

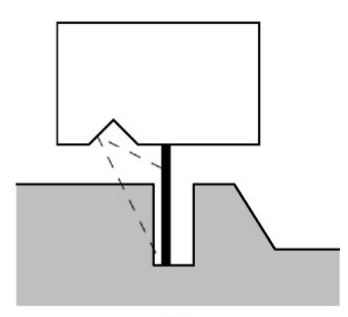

(c)

Fig.4: The two types of occlusions :(a) system; (b) laser occlusion; (c) camera occlusion 
System used is a CCD camera and a laser which are coupled and form a fixed sensor unit (Fig.5). The laser beam is spread into an illuminating plane. The illuminating plane intersects with the object surface, forming a planar curve (laser stripe). The range image is obtained by scanning the scene with a series of parallel illuminating planes. This can be achieved by moving the origin of the camera-laser sensor unit in a plane called the scanning plane. This scanning plane is orthogonal to the illuminating plane.

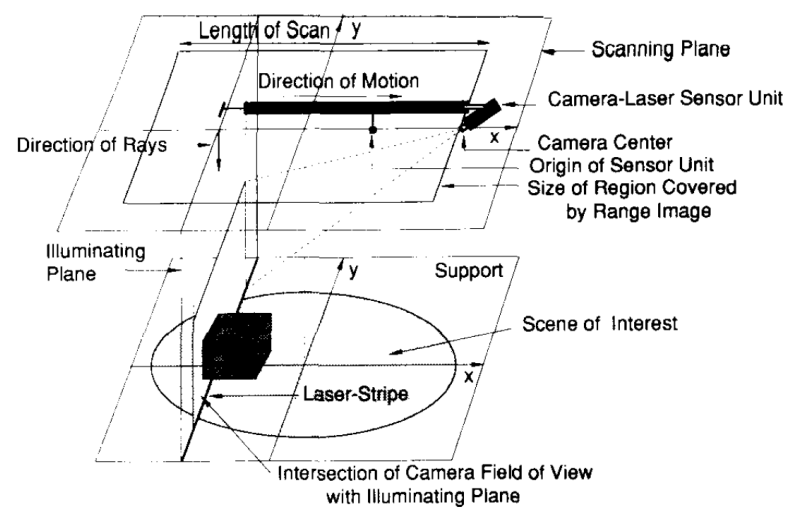

Fig.5: The sensing system used by Maver \& al.

Maver and Bajcsy use sensor planning during scene modeling. An initial image is acquired from an arbitrary direction. The occluded areas in this image (range shadows) are determined and approximated as polygons. Each contour of the range shadows is segmented into a series of straight lines. The breakpoints of the contour segmentation are used to divide the occluding polygons into areas. Then, viewing angles for every pixel in the occluding polygons are calculated. A viewing angle is the sector containing all scanning directions from which a pixel is visible. For each area of the occluding polygons, the histogram of viewing angles is calculated. The histogram shows how many pixels can be seen from a certain direction. Histogram decomposition is then performed to find the next scanning direction; the next view direction is selected from the intervals defined by the maxima in the final histogram.

In [23], Pito \& al. chose occlusion edges as a mechanism for the NBV search. They observed that the void volume can be represented by defining only the void surface near edges of the current model, which they represented by small rectangular patches attached to occluding edges of the seen surface. In general, the portion of the shdow nearest the occlusion boundary will be nearest to the real object surface and therefore be the best region to search next. Furthermore, such regions fit the overlap constraint. Pito's NBV algorithm used an intermediate space representation, "positional space" (PS), as a repository for two types of visibility information - object surface visibility and sensor scanning potential. A virtual positional space surface (PSS), with a shape appropriate to the sensor-positioning system combination, was placed between the object and the sensor workspace. Object surface space (represented as a mesh), PSS, and viewpoint space were discretized (Fig.6).

The visibility of each triangular mesh element on the object surface can be encoded in positional space by tracing an "observation ray" from the mesh element to a PSS cell. The direction of an unoccluded observation ray relative to a local frame of reference on the PSS can be specified by two angles, termed the positional space direction (PSD). Pito chose to encode surface visibility as an analogue value equal to the area of the surface element visible by a given ray weighted by a confidence measure tied to the measurement grazing angle. Thus, PS was a scalar field in four dimensions $\mathrm{P}(\mathrm{u}, \mathrm{v}, \mu, \mathrm{A})$ where $\mathrm{u}, \mathrm{v}$ were coordinates in PSS and $\mu$, Á were the components of PSD. Encoding the image of both the seen surface and void patches in PS provided a means to apply an overlap constraint between views to meet registration and integration requirements. The range camera's scanning potential at a given viewpoint can be similarly encoded in positional space by determining the intersection of "ranging rays" from the optical transmitter and receiver with the PSS. A separate image was calculated for each viewpoint. Using PS as a placeholder for ranging and observation rays facilitated determining which of them were collinear, as well as aiding the application of NBV constraints 


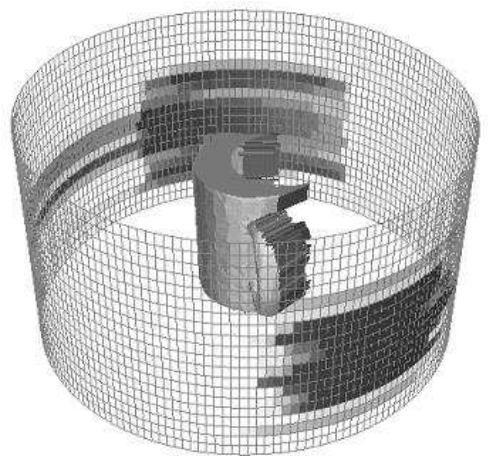

(a)

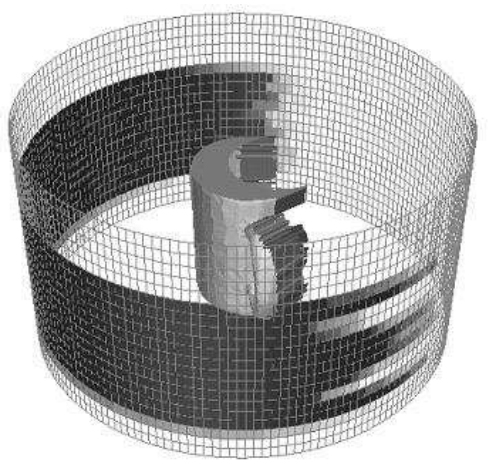

(b)

Fig.6: Range image inside the PSS. Each cell of the PSS is colored with the cumulative confidence of the observation rays (a) of void patches which project into that cell and (b) of faces of the model which project into that cell.

The first methods (model-based method) are suitable when CAD model of the object is available. This kind of sensor planning has been developed for several different purposes, for example object recognition, general robot vision tasks, inspection, .... The second methods (non-based model method) allows viewpoint planning without a priori knowledge on the shape. They are divided in two categories: the volume based methods ([19], [20], [21]) and the surface based methods ([22], [23], [24]). A summary of these methods is shown table 1

\begin{tabular}{|l|c|c|c|}
\hline & \multirow{2}{*}{$\begin{array}{c}\text { Model- } \\
\text { based method }\end{array}$} & Surface & Volume \\
\cline { 3 - 4 } & & & \\
\hline Cowan [17] & $\mathrm{X}$ & & \\
\hline Scott [18] & $\mathrm{X}$ & & $\mathrm{X}$ \\
\hline Connolly [19] & & & $\mathrm{X}$ \\
\hline Banta [20][21] & & & \\
\hline Maver [22] & & $\mathrm{X}$ & \\
\hline Pito [23] & & $\mathrm{X}$ & \\
\hline Yuan [24] & & $\mathrm{X}$ & \\
\hline
\end{tabular}

Table 1: Summary of the described method

The majority of these algorithms leads to good results in the case of simple shapes, like mechanical parts, or convex objects. As yet, view planning for high-quality complex object reconstruction has no general-purpose solution and maybe such solution never exist. That's why our objective is not the exhaustive digitization of an object, but the most complete possible surface coverage.

As we plan to produce 3D models of cultural heritage artefacts, only non-based methods are applicable, due to the complexity of the shape. CAD model is not available and methods with a first coarse acquisition can be used but the first phase must be done manually to correctly adjust the number of views necessary. In non-model based methods, surface based methods enables to work directly on 3D shape data acquisition, instead of volume based methods that require a voxel space management in addition, which implies an important computing cost. Our proposed method is inspired from work presented in [22], [23], [24] and is described in section 3 and 4, and is summarized at Fig. 7.

The first phase of the proposed method is based on the approach developed by Yuan \& al [24] and uses Mass Vector Chain to determine the next best view. It leads to a maximum surface coverage with remaining holes due to self-occlusions. This method is simple to implement and requires very small computing time. 
The second phase computes the position and the orientation of the next view, by using remaining holes in mesh. This phase is based on the methods developed by Maver \& al [22] and Pito [23].

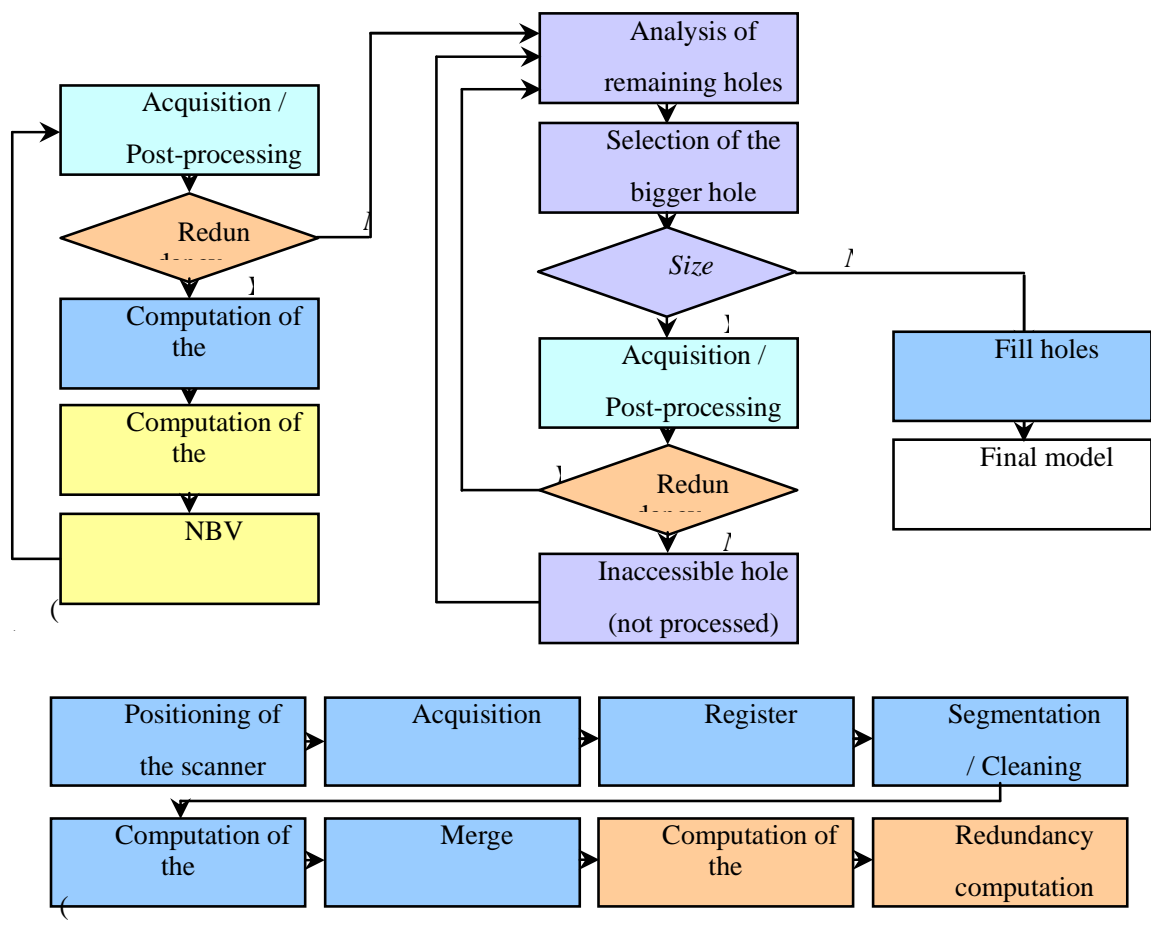

Fig.7: The proposed method is in two steps (a): the first step allows to create a coarse model with MVC, and the second uses "holes" to complete the unprocessed surface. For each new view point, acquisition and post-processing are carried out (b)

\section{Mass Vector Chain (MVC)}

MVC allows to estimate a view direction from surface patch. In our case, each face is considered as a surface patch.

By definition (Fig.8), a mass vector chain of an object is a serie of weighted vectors. In this serie, a vector $\vec{V}_{i}$, is assigned to each individual surface patch $S_{i}$ of the object. This vector points to the average normal direction $\vec{n}_{i}$, of the surface patch $S_{i}$ and its weight is the projected region $R_{i}$ on a plane $P_{i}$ perpendicular to $\vec{n}_{i}$

$$
\vec{V}_{i}=\vec{n}_{i} R_{i}
$$

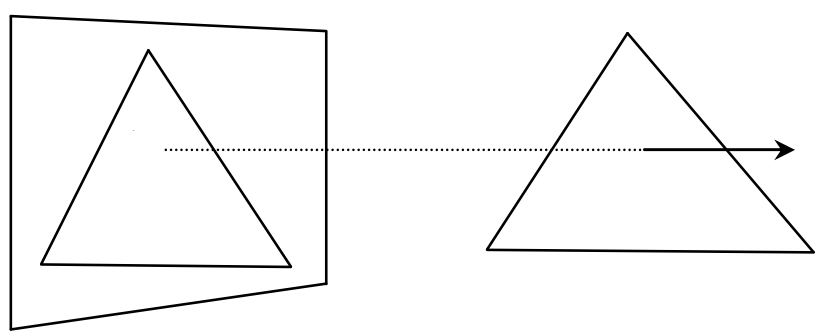

Fig.8: Definition of mass vector

For the surface patch, $\vec{n}_{i}$ is its average visible direction and $R_{i}$ is the surface size when viewed in that direction. It has been demonstrated [24] that, for an object of convex surfaces, the total Gaussian mass of the surfaces must be zero, 


$$
\iint_{s} G(n(s)) n(s) d s=\oiint n(s) d s=0
$$

where $G(n(s))$ is the Gaussian mass with a same $n(s)$. This conclusion also applies to ordinary objects. Since the total Gaussian mass of an object is the sum of subtotals of individual surface patches, (2) can be further grouped in terms of surfaces.

$$
\oiint n(s) d s=\sum_{j=0}^{m-1} \iint_{s j} n(s) d s=0
$$

On the other hand, the total mass vector of an object is the sum of all its mass vectors, i.e.

$$
\sum_{j=0}^{m-1} \vec{V}_{j}
$$

The total mass vector can be derived. The result points out that the boundary surfaces of an object compose a closed surface boundary only when their mass vectors form a closed chain.

$$
\sum_{j=0}^{m-1} \vec{V}_{j}=\sum_{j=0}^{m-1} \iint_{s i} n(s) d s=0
$$

The mass vector sum of a closed object model is a zero vector. If, during reconstruction, the total of all mass vectors of a building model is a no zero vector $\vec{V}_{d i r}$, there must be some unprocessed surface patches whose mass vectors sum to be the negative of $\vec{V}_{d i r}$. If the number of processed surface patches is $m$ ', then, from the relation

$$
\begin{aligned}
& \sum_{j=0}^{m^{\prime}-1} \vec{V}_{j}+\sum_{j=m^{\prime}}^{m-1} \vec{V}_{j}=0 \\
& \text { we obtain } \sum_{j=m^{\prime}}^{m-1} \vec{V}_{j}=-\sum_{j=0}^{m^{\prime}-1} \vec{V}_{j}=-\vec{V}_{d i r}
\end{aligned}
$$

Defined to be the average normal $\vec{n}_{i}$, each mass vector $\vec{V}_{i}$, is actually the average visible direction of that surface patch. Therefore, $\vec{V}_{\text {dir }}$ provides an estimated direction from which these unprocessed surface patches could be observed.

This can be further explained by the surface represented in Fig. 9 for convex surfaces. Since a convex object has a unique extended Gaussian image expression [24], a Gaussian sphere [25] covering the object stands for its boundary condition, or a circle in a two-dimensional case. Suppose four surface patches are extracted (Fig.9a); the mass vector sum is $\vec{v}=\vec{v}_{0}+\vec{v}_{1}+\vec{v}_{2}+\vec{v}_{3} \neq \overrightarrow{0}$ and give the view direction for the next view (Fig.9b and 9c). An example of MVC is shown in Fig.10.
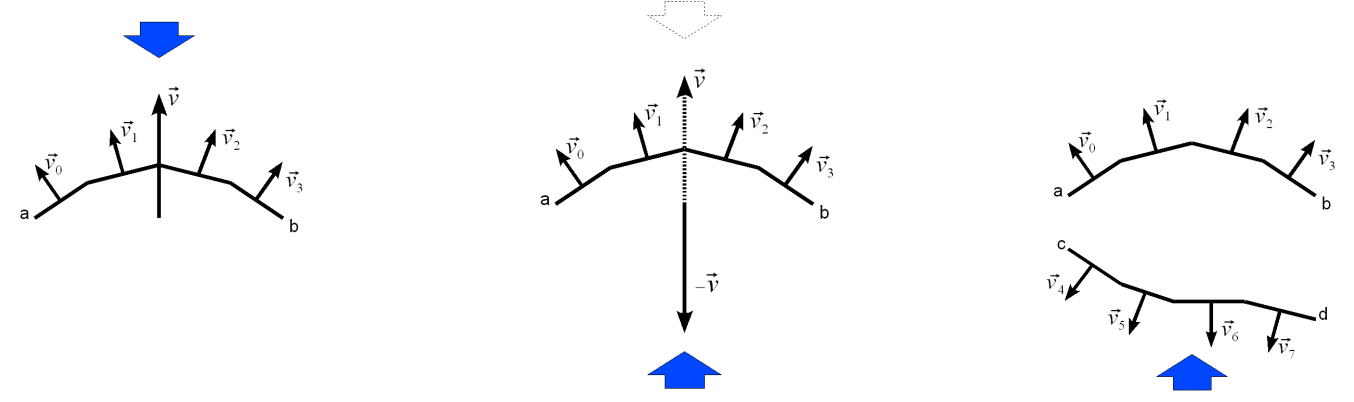

Fig.9: View estimation of processed faces (a) and unprocessed faces (b), and new acquisition (c) 
A similar Gaussian sphere can also be used to examine the boundary of concave surfaces. Surfaces must be extracted to identify concave and convex surface. But here, no surface is extracted, there is no differentiation between concave and convex shape, because MVC are used only to deliver a coarse model.

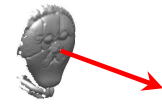

$\leftarrow$ (a)

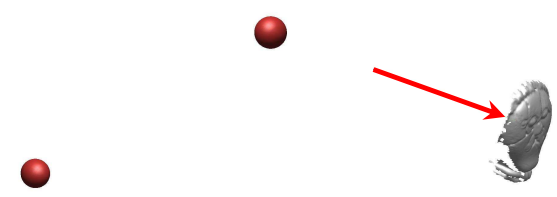

(b)

Fig.10: Red sphere represents the scanner position. MVC is calculated (a) and gives direction for the next view (b)

\section{Proposed solution for automatic acquisition and post-processing}

To test different algorithms without dealing with the mechanical constraints, we implemented algorithms in a simulated scanner. The simulated scanner uses raytracing to simulate a triangulation laser scanner. 3D model of the object is needed. The procedure is as follows:

- the scanner is positioned manually for the first position

- the user defines the parameters of the scanner : resolutions in $\mathrm{X}$ and $\mathrm{Y}$ and angles of view (horizontally and vertically).

- the algorithm carries out raytracing: it calculates the intersection of each ray with the 3D model [26].

- A point clouds is created and then triangulated [27].

Simulated algorithms were then implemented on a positioning system. Before beginning, we assume that the object respect the following constraint :

- the field of view constraint : the object must be entirely in the field of view of the scanner

- the material constraint : the surface object can be digitized (not transparent or reflective)

- the positioning constraint : the size of the object must be adapted to the positioning system

The proposed method is divided in two steps and is a surface based-method. The first step is the acquisition of a 3D coarse model: a range image is acquired from a position chosen arbitrarily, then MVC is computed to determine the next position and the 3D scanner is moved consequently. The MVC method previously described is capable only of estimating viewing direction, not position. Then, for each new acquisition, the scanner is directed towards the center of mass of previous acquisitions, at a present distance (calibrated from the object size). Information of position of the 3D scanner allows to readjust each scan in the same coordinate system (registration). Each scan is segmented (object-environment) in related components (Fig.11). The largest components are selected until the sum of their surface is more than $80 \%$ of the surface of the view. This threshold has been determined experimentally. We assume that the object is centered and occupies the major part of the field of view. If the threshold is higher than $80 \%$, the quality of the model will be degraded. If it is lower than $80 \%$, the number of views required will be more important.
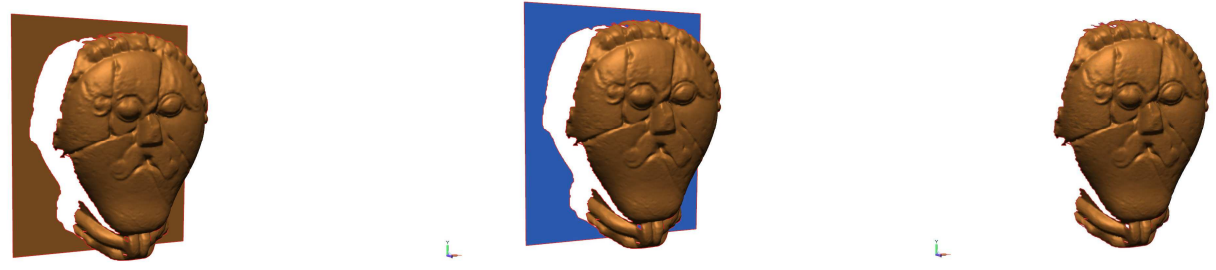

Fig.11: Segmentation of the view in related components 
Then, the view is cleaned [28]: all the abnormals faces (three faces with one same edge, spikes, ...Fig.12) are removed. Besides, all the points of which the angle of view is higher than a given angle $\left(\approx 60^{\circ}\right)$ are also removed. If the new view don't contribute to decrease the size of unprocessed surface, this step ends.

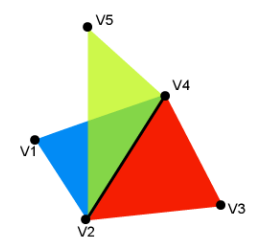

(a)

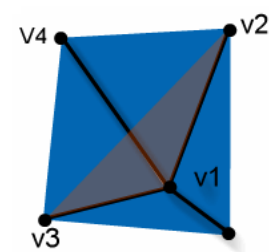

(b)

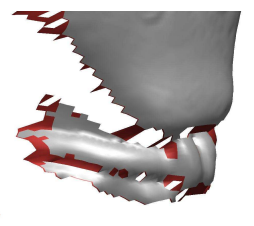

(c)

Fig.12: Post-processing : abnormals faces (a), crossing faces (b) and spikes (c) are removed.

In the second step, all the boundary loops ("holes") remaining in the 3D model are selected. This step is inspired of [22] and [23], but the implementation is innovative. Here, a hole shows a lack of faces, whereas a physical hole has a surface. The detection of the holes is done by locating the edges of the model which belong only to one face. For each hole thus found, its "normal", its "position" and its "size" is calculated (Fig.13)

The "position" $P_{h}$ corresponds to the "center of mass" of the hole, obtained starting from the $m$ vertice $P_{i}$ composing the hole.

$$
P_{h}=\frac{1}{m} \sum_{i=1}^{i=m} P_{i}
$$

The "normal" $\vec{V}_{h}$ is given by totaling the normals $\vec{V}_{i}$ of the vertice which form the hole.

$$
\vec{V}_{h}=\sum_{i=1}^{i=m} \vec{V}_{i}
$$

The "size" $D$ of the hole corresponds to the maximum distance between the "center of mass" $P_{h}$ and the points which form the hole

$$
D=\max _{i}\left(\left|P_{i} P_{h}\right|\right)
$$

These three parameters allow to calculate the next position: the scanner is directed towards the center of the hole,

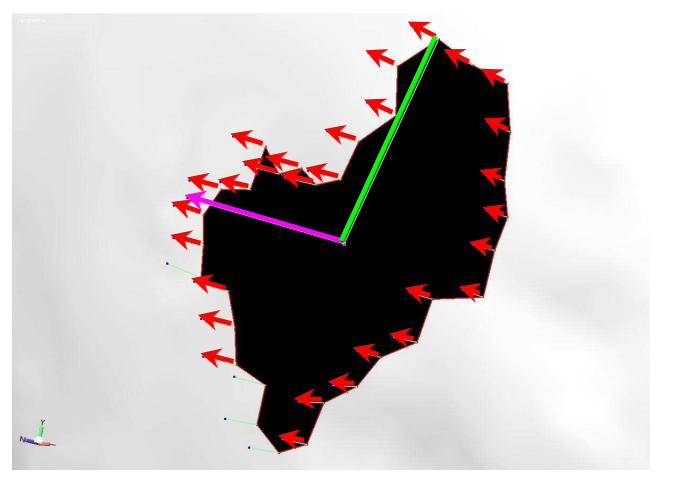

Fig.13: Holes features : normal, size, position

according to a direction reverses with the normal of the hole, if there is not self-occlusions. Else, a Gaussian sphere [13] is defined, corresponding to a "sphere of positions" (Fig.14). For each points of hole, positions are tested, and the position from which the most of points are visible is selected for the next view. 

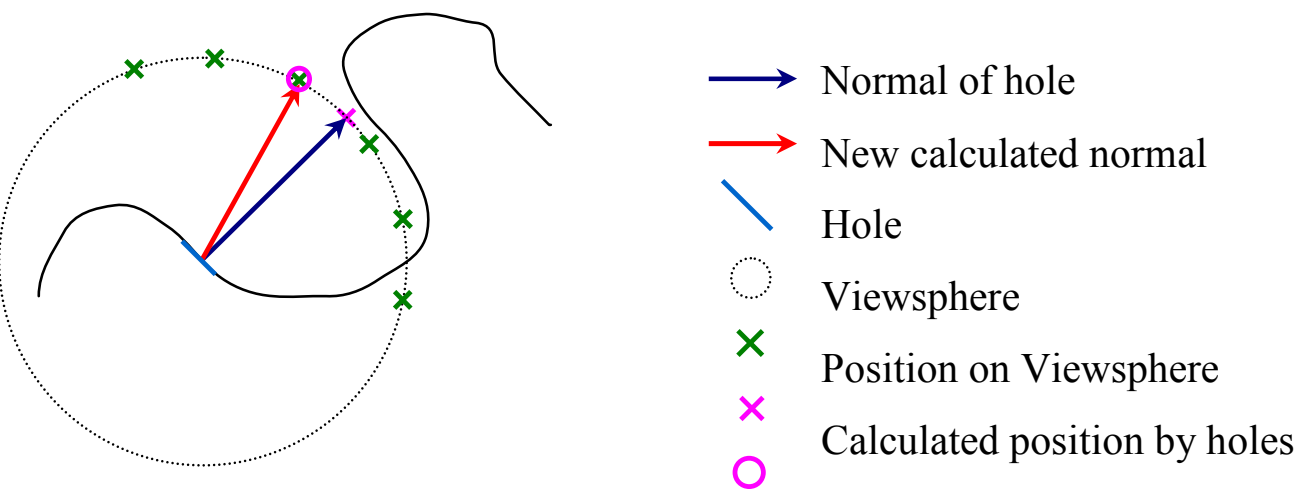

Fig.14: Viewsphere for self occlusions

Then, new 3D view is segmented before merging: faces corresponding to the hole are only preserved: on the new 3D view, all the points $P_{i}$ which verify $\left|P_{i} P_{h}\right|>D$ are removed (Fig.15). D is defined in equation 10.

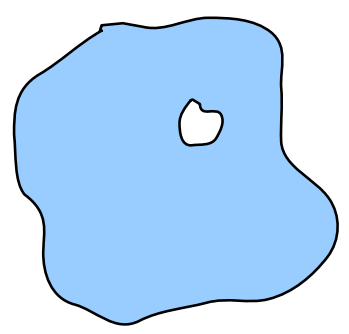

Object

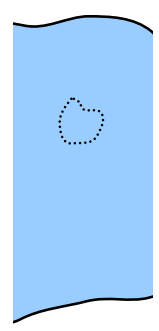

New view

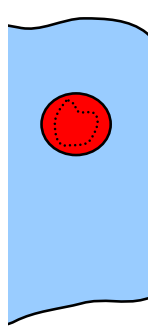

Segmentation

Fig.15: Segmentation of the new view. The redundant data is removed for each new acquired view.

\section{$5 \quad$ Results}

Proposed system on which algorithms has been tested uses a motion stage four axes and a rotative stage, on which a scanner is set, allowing five degrees of freedom (Fig. 16-17). This system provides travel range from $1370 \mathrm{~mm}$ ( $\mathrm{X}$ axis), $990 \mathrm{~mm}$ ( $\mathrm{Y}$ axis), $440 \mathrm{~mm}$ ( $\mathrm{Z}$ axis), 320 ( $\alpha$ axis) and $360^{\circ}$ ( $\beta$ axis). The used scanner is a laser triangulation-based device (Konica Minolta Vi910 [14]). To illustrated our results, studied artefacts is a small head $(95 \times 75 \times 35 \mathrm{~mm})$ shown in Fig. 18a.
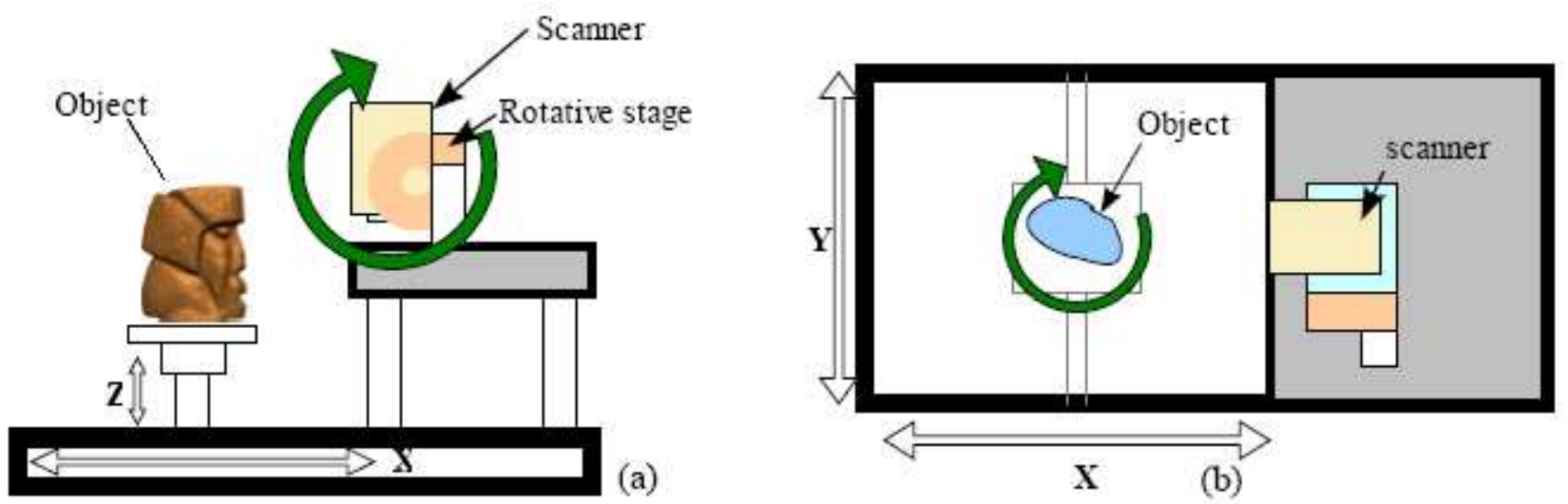

Fig.16: Proposed system: (a) schematic front view, (b) schematic top view 


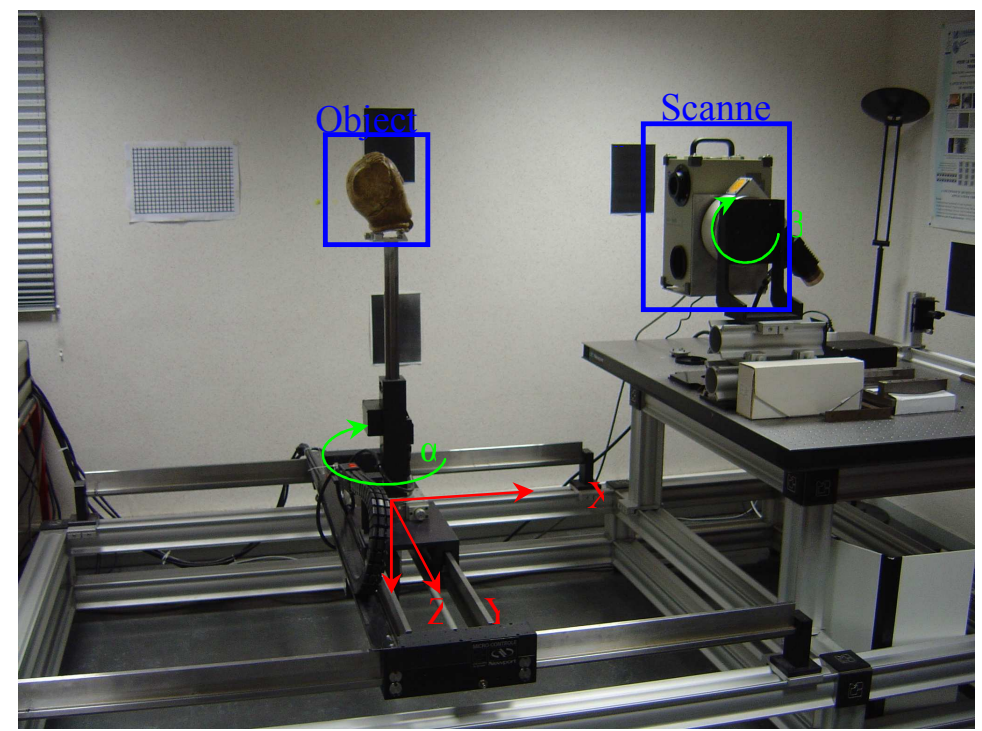

Fig.17: Proposed system

First position is selected in order to fit the field of view on the studied object (Fig. 18b). Acquisition is performed from this position (Fig. 18c) and then MVC is calculated and a new direction is determined (Fig. $18 \mathrm{~d})$. The system moves to place the object and the scanner in the new position. New view is acquired (Fig.18e) and merged with the first view (Fig. 18f), after being cleaned (segmentation object-environment, delete abnormals faces, ...). Fig. 18h and Fig. 18i shows the 3D model before and after acquisition of the third view. This procedure is repeated until the stop criterion is reach. This criterion is calculated from the views surface. If the redundant surface between the 3D model without the new view and the 3D model with the new view is greater than $90 \%$, this step ends.

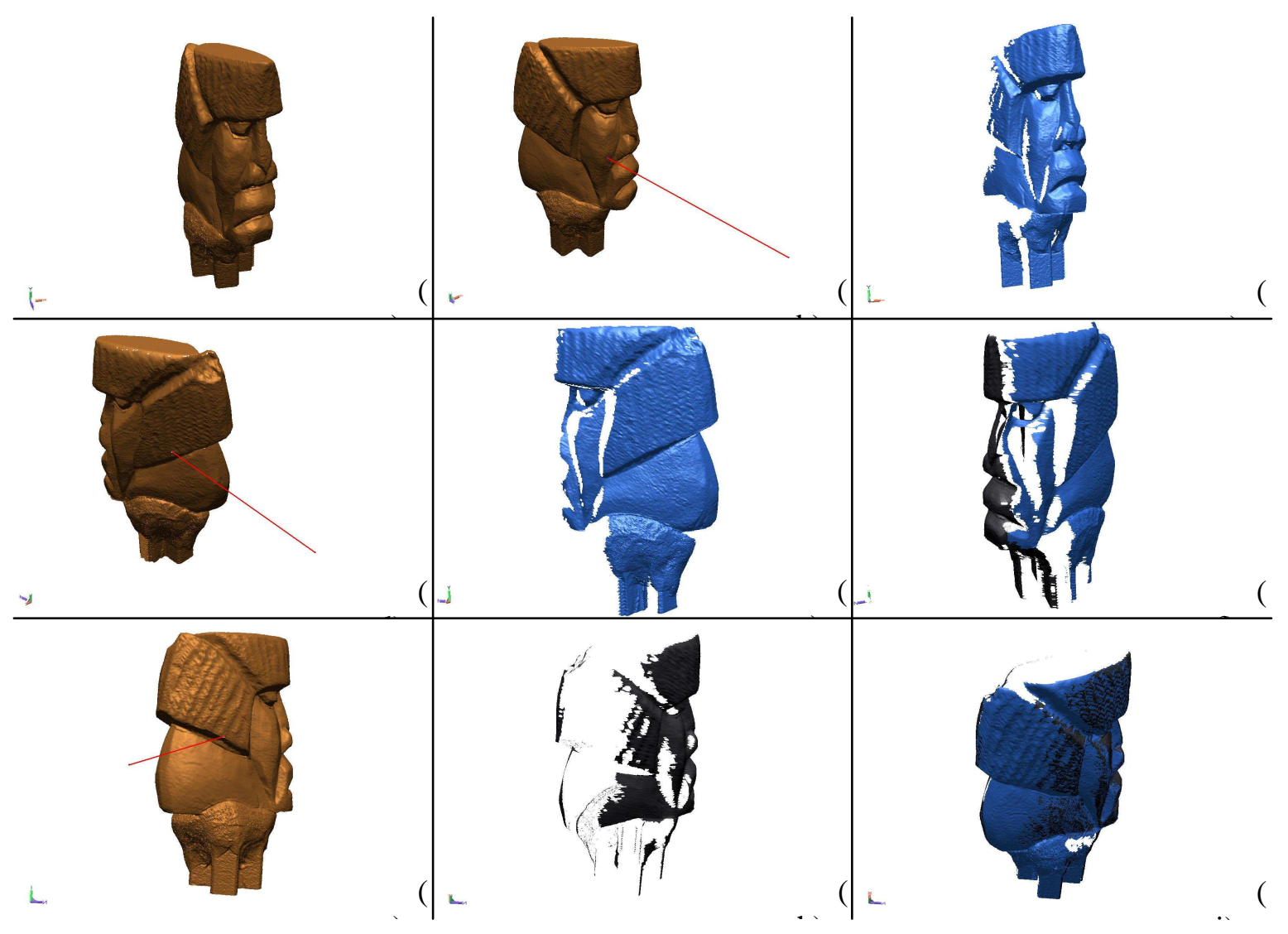

Fig.18: Studied artefact (a), and different steps of MVC 
In this example, MVC allows the capture of six views (only three are represented in Fig. 18). The obtained 3D model is shown in Fig. 19(a).
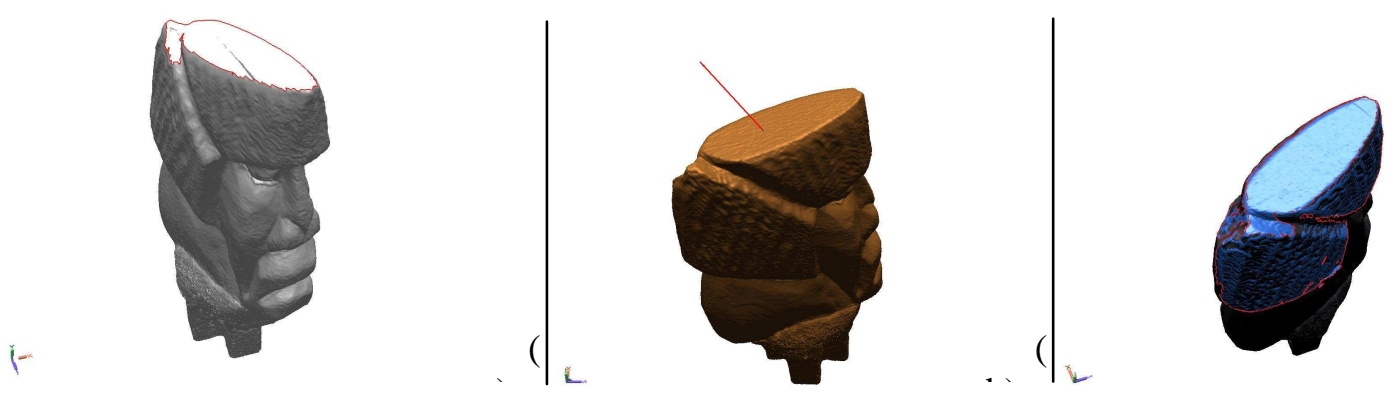

Fig.19: 3D model obtained with the MVC (a); New direction from $2^{\text {nd }}$ phase (b) and new view (c).

In this model, two remaining holes are present, with two opposite "normals": one on the top of the model, and one below, where the object is set. The second hole is ignored (acquisition is impossible). The features of the first hole is calculated (position, normal, size), and a direction is found (Fig.19). New view is segmented and redundant data are removed (Fig.12).

Proposed solution allows to obtain automatically complete 3D model of the studied artefact with seven views in $10 \mathrm{~min}$. Proposed system allows to move object and scanner with five degrees of freedom. This system is well adapted for small objects $(100 \times 100 \times 100 \mathrm{~mm})$ but not for larger objects: travel range may be insufficient and the lower part of the object isn't visible. For these objects, methods must be implemented on others systems, like CMMs or robotized arm. In simulation, several objects are tested (cultural heritage artefacts and mechanical parts). Some results are shown in table 2 and fig. 20 . To validate our method, we calculate the number of views in the two phases, the average resolution obtained and the percentage of surface coverage.

\begin{tabular}{|l|c|c|c|c|}
\hline & $\begin{array}{c}\text { Number of views } \\
\text { Phase 1 }\end{array}$ & $\begin{array}{c}\text { Number of views } \\
\text { Phase 2 }\end{array}$ & $\begin{array}{c}\text { Resolution } \\
(\mathrm{mm})\end{array}$ & $\begin{array}{c}\text { Coverage } \\
\text { surface (\%) }\end{array}$ \\
\hline Ammonite & 5 & 1 & 1,23 & 99 \\
\hline Frog & 6 & 3 & 1,26 & 100 \\
\hline Wheel rim & 6 & 9 & 1,31 & 57 \\
\hline Mzeke & 7 & 1 & 1,19 & 100 \\
\hline Niepce bust & 5 & 13 & 1,28 & 100 \\
\hline Painter bust & 5 & 7 & 1,22 & 99 \\
\hline Valve & 11 & 6 & 1,26 & 99 \\
\hline Shrek & 5 & 14 & 1,22 & 100 \\
\hline Statue & 7 & 38 & 1,28 & 98 \\
\hline Saint Jean statue & 8 & 37 & 1,25 & 97 \\
\hline Virgin statue & 3 & 11 & 1,21 & 68 \\
\hline Mug & 4 & 2 & 1.20 & 99 \\
\hline Bone & & & & 93 \\
\hline
\end{tabular}

Table 2: Results for several objects: number of views in phase 1 and 2, resolution and digitized surface 
Objects can be classified in three categories:

- convex object : ammonite, frog, Mzeke, Shrek, bone.

- globally convex object with cavities and self-occlusions: statues, busts, valve.

- concave objects : wheel rim, mug.

For the convex object, the percentage of surface coverage is superior to $98 \%$ and the number of views required is low. For more complex object, the percentage of surface coverage is higher than $97 \%$ but the number of views is important because of occlusions. However, the number of views obtained is comparable to the number of acquisitions that would do an experienced operator (for example, valve requires 16 views). But for concave objects, the percentage of surface coverage is lower than $80 \%$.

The different segmentation and post-processing apply on the 3D views allow to control the quality of the 3D model, and the average resolution obtained corresponds to the resolution of the scanner.

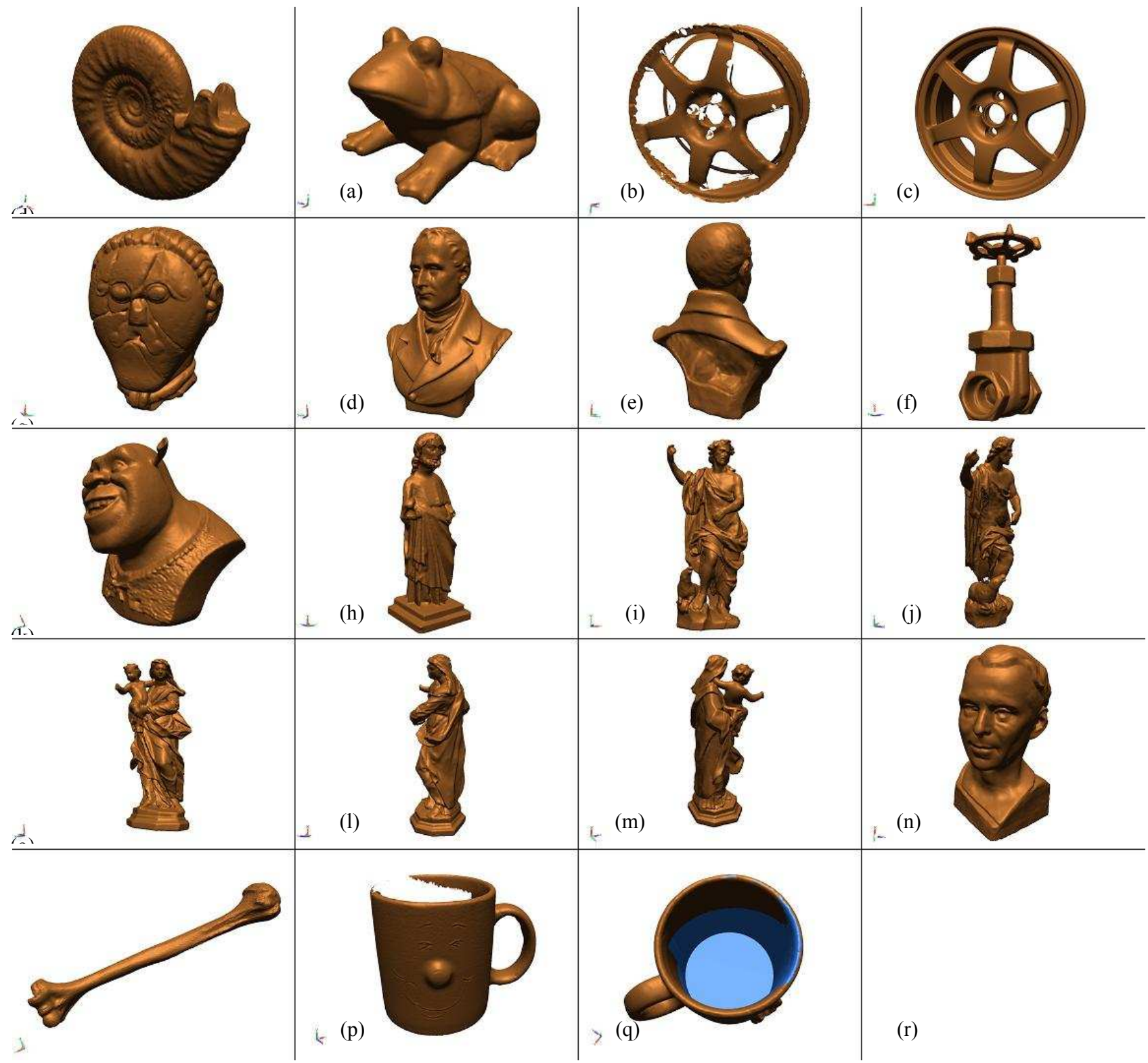

Fig.20: Results for different objects : ammonite (a), frog (b), wheel rim (c), original wheel rim (d), Mzeke (e), Niepce bust (e-f), valve (g), shrek (h), statue (i), Saint Jean (j-k), Vierge (1-n), painter bust (o), bone (p), mug (q-r) 


\section{Conclusion}

In this paper, a simulation tool is introduced to simulate the acquisition of 3D models. This tool allows to simulate different NBV (Next-Best-View) solutions and post processing.

NBV methods have been studied and the method we selected is a multi-phase approach. The first phase is based on Mass Vector Chains to solve NBV problem. This phase is able to predicate the viewing direction of unprocessed surfaces and allow to determine viewpoint for the next acquisition. At the end of this step, a coarse model is created, with remaining holes. From analysis of holes in the mesh, the second phase calculates the best next viewpoint to acquire the missing data. The algorithm is self-terminating and allows to obtain automatically - without any user intervention - a complete 3D model of an object. Besides, thanks to the different post-processing and segmentation, quality of the mesh is controlled. But for globally concave object, the method does not work.

Future work concerns the application of these methods to various 3D scanners. We plan to demonstrate that our method is applicable to different 3D measurement devices available in our research team (time-offlight system, laser triangulation, ...). The algorithms can be reused by adding constraints on various parameters (field of view, resolution, workspace).

We also want to use this method with different positioning systems equipped with a new 3D sensor. A new prototype based on a CMM (Coordinate Machine Measurement) is under development and will allow better results thanks to the increased number of degree of freedom. A new starting collaboration with industrial partners will enable to acquire a robotized arm also equipped with a 3D scanning head. We will soon demonstrate with this new equipment that our algorithms are applicable for 3D reconstruction of numerous collections of cultural heritage artefacts.

\section{Acknowledgement}

The author would like to thank Dynamic3D company [29] for funding this project.

\section{References}

[1] P. J. Neugebauer, "Scanning and Reconstruction of Work Pieces from Range Images," in Second IFIP 5.10 Workshop on Virtual Prototyping, Automation and Robotics Research Institute, Arlington, Texas, May 6-8, 1996

[2] T.T. Allard, "The Role of 3D Printing in Biological Anthropology", phD Thesis, University of Manitoba, Winnipeg, 2006

[3] M. Friess, L.F. Marcus, D.P. Reddy, E. Delson, "The Use of 3d Laser Scanning Techniques for the Morphometric Analysis of Human Facial Shape Variation", XIV International Congress of Prehistoric and Protohistoric Science, Liège (Belgium), September 2001.

[4] B. Mafart, G. Guipert, M.A. de Lumley, G. Subsol, "Three-Dimensional Computer Imaging of Hominid Fossils a New Step in Human Evolution Studies", Can Assoc Radiol J - 2004 Oct;55(4):26470

[5] X. Li, J. Feng, H. Zha, "3d Modelling Of Geological Fossils and Ores by Combining High-Resolution Textures with 3d Scanning Data", Proceedings of Ninth International Conference on Virtual Systems and MultiMedia 2003, Montreal, Canada, October 2003 - VSMM2003, pp.686-693.

[6] Y. Ge, "Noncontact 3D Biological Shape Measurement from Multiple Views", Master's thesis, Dept. of Computer Science, The Univ. of Western Australia, 1994.

[7] M. Levoy, K. Pulli, B. Curless, S. Rusinkiewicz, D. Koller, L. Pereira, M. Ginzton, S. Anderson, J. Davis, J. Ginsberg, J. Shade, D. Fulk, "The digital michelangelo project: 3D scanning of large statues", In Computer Graphics, SIGGRAPH 2000 Proceedings, 131-144.

[8] B. Loriot, Y. Fougerolle, R. Seulin, C. Sestier, "3D acquisition and modeling for flint artefacts analysis", SPIE Optical Metrology - Optics for Arts, Architecture, and Archaeology, Munich, Allemagne, June 2007. 
[9] R. Seulin, C. Stolz, D. Fofi, G. Millon, F. Nicolier, "3D Tools for Ancient Wooden Stamps Analysis and Conservation", The Imaging Science Journal, Maney Publishing, 54(2):111-121, June 2006

[10] W. Böhler, M. Bordas Vicent, G. Heinz, A. Marbs, H. Müller, "High quality scanning and modeling of monuments and artifacts", Proc. FIG Working Week 2004, Athens, Greece, 22-27 May

[11] S. El-Hakim, A. Beraldin, M. Picard, "Detailed 2D Reconstruction of Monuments using Multiple Techniques", In: ISPRS/CIPA International Workshop on Scanning for Cultural Heritage Recording, Corfu, Greece, 58-64

[12] W. Boehler, A. Marbs "3D Scanning Instruments", Proceedings of the CIPA ISPRS International workshop on Scanning for cultural heritage recording, Corfou, Greece, September 1-2, 2002

[13] B. Curless, "From Range Scans to 3D Models", Computer Graphics, 33(4), november 1999.

[14] Konica-Minolta, www.konicaminolta.com/instruments/products/3d/index.html,

[15] F. Prieto, "Métrologie Assistée par Ordinateur : Apport des Capteurs 3D sans Contact", phD Thesis, INSA Lyon, 1999

[16] W.R. Scott, G. Roth, J.-F. Riverst, "View Planning for Automated Three-Dimensional Object Reconstruction and Inspection", ACM Computing Surveys, vol. 35(1), pp. 64-96, 2003

[17] C.K. Cowan, P.D. Kovesi, "Automatic Sensor Placement from Vision Task Requirements", IEEE Trans. Pattern Anal. Mach. Intell., vol. 10(3), pp. 407-416, 1998

[18] W.R. Scott, G. Roth, J.-F. Riverst, "View Planning for Multistage Object Reconstruction", In $14^{\text {th }}$ Int. Conf. on Vision Interface, Ottawa, pp. 64-71, june 2001

[19] C.I. Connolly, "The Determination of Next Best Views", In Proc. IEEE International Conference on Robotics and Automation, IEEE Computer Society Press, 432-435, 1985

[20] J.E. Banta, Y. Zhien, X.Z. Wang, G. Zhang, M.T. Smith, M.A. Abidi, “A Best-Next-View Algorithm for Three-Dimensional Scene Reconstruction Using Range Images", In Computer Graphics (SIGGRAPH'87 Proceedings) SPI Intelligent Robots and Computer Vision XIV. Algorithms, Techniques, Active Vision and Material Handling, Philadelphia, PA, 1995

[21] J.E. Banta, L.M. Wong, C. Dumont, M.A. Abidi, "A Next-Best-View System for Autonomous 3-D Object Reconstruction", Systems, Man and Cybernetics, Part A, IEEE Transactions on, 2000

[22] J. Maver, R. Bajcsy, "Occlusions as a Guide for Planning the Next View", IEEE, Transactions on Pattern Analysis and Machine Intelligence, 15(5), may 1993

[23] R. Pito, "A Sensor Based Solution to the Next Best View Problem", International Conference on Pattern Recognition, 941-945, 1996

[24] X. Yuan, “A Mechanism of Automatic 3D Object Modeling”, IEEE, Transactions on Pattern Analysis and Machine Intelligence, 17(3), march 1995

[25] B.K. Horn, "Extended Gaussian images", Proc. IEEE, 72:1656-1678, 1984.

[26] R.A. Goldstein, R. Nagel, “3D Visual Simulation”, Simulation, 16(1):25-31

[27] J.-D. Boissonnat, B. Geiger, "Three-dimensional reconstruction of complex shapes based on the Delaunay triangulation", Proc. SPIE Biomedical Image Processing and Biomedical Visualization, 964975, july 1993

[28] T. Weyrich, M. Pauly, S. Heinzle, R. Keiser, S. Scandella, M. Gross, "Post processing of scanned 3d surface data", In Symposium on Point-Based Graphics, 85-94, 2004.

[29] Dynamic3D Company, 3D acquisition and post-processing, http://www.dyn3d.com 